\title{
Prevalência de distúrbios do sono na pós-menopausa
}

\author{
Sleep disturbance prevalence in postmenopausal women
}

\author{
Helena Hachul de Campos $^{1}$, Lia Rita Azeredo Bittencourt ${ }^{2}$, Mauro Abi Haidar ${ }^{3}$, \\ Sérgio Tufik ${ }^{4}$, Edmund Chada Baracat ${ }^{5}$
}

\section{RESUM0}

Objetivo: avaliar a prevalência das queixas de distúrbios do sono pela polissonografia em amostra de mulheres na pósmenopausa. Métodos: foram selecionadas 33 mulheres na pós-menopausa com média de idade de 56 anos, índice de massa corporal médio de 27, tempo de pós-menopausa de 7,7 anos e índice de Kupperman de 17. Adotaram-se os seguintes critérios de inclusão: idade entre 50 e 65 anos, no mínimo um ano de amenorréia e FSH plasmático superior ou igual a $30 \mathrm{mU} /$ $\mathrm{mL}$, sem uso de terapia hormonal prévia e exames laboratoriais normais. Foram excluídas as pacientes com doenças clínicas graves e/ou descompensadas, suspeita de câncer de endométrio e/ou mama; índice de massa corporal maior ou igual a $30 \mathrm{e}$ uso de hipnóticos. As pacientes responderam a questionário específico contendo perguntas sobre as características do sono e foram submetidas a polissonografia completa durante uma noite inteira. Foram calculadas separadamente as freqüências em porcentagens das queixas de sono e dos diagnósticos polissonográficos. Resultados: a prevalência de insônia subjetiva foi $61 \%$, sendo que na polissonografia foi de $83 \%$. A queixa de apnéia foi registrada em $23 \%$ e, na polissonografia, em $27 \%$. A prevalência subjetiva de movimentos periódicos de pernas foi de $45 \%$ e a objetiva foi de $27 \%$. Conclusão: houve alta prevalência de distúrbios do sono na pós-menopausa, em especial de insônia, apnéia e de movimentos periódicos das pernas. Nesta fase da vida, ocorre piora da qualidade do sono.

PALAVRAS-CHAVE: Pós-menopausa; Distúrbios do sono; Distúrbio do início e da manutenção do sono; Síndromes da apnéia do sono; Síndrome das pernas inquietas; Polisonografia

\section{ABSTRACT}

Purpose: to evaluate the prevalence of reported sleep disturbances through polysomnographic recording (PSG) in a sample of postmenopausal women. Methods: thirty-three postmenopausal women with a mean age of 56 years, a mean body mass index (BMI) of $27 \mathrm{~kg} / \mathrm{m}^{2}$, with 7.7 years of recognized postmenopausal period, and a mean Kupperman index of 17, were selected. The inclusion criteria were: age range from 50 to 65 years, at least one year of amenorrhea and an FSH which equaled or exceeded $30 \mathrm{mU} / \mathrm{mL}$; they should not be undergoing hormone therapy, and should display normal laboratory test results. The patients with severe clinical diseases and/or decompensated were excluded; also the ones with suspicion of carcinoma of endometrium and/or breast cancer, a BMI over $30 \mathrm{~kg} / \mathrm{m}^{2}$ and those who ingested hypnotic drugs. The patients followed a routine climacteric check-up, answered a questionnaire about sleep and underwent an all-night PSG recording. Frequencies in percentage of emerging sleep complaints based on the questionnaire and those pertaining to PSG diagnosis were then calculated separately. Results: the subjective prevalence of insomnia was $61 \%$ against $83 \%$ in the PSG recordings. The prevalence of apnea reported was $23 \%$ against $27 \%$ in the PSG. The subjective restless legs syndrome prevalence was $45 \%$, and the objective, $27 \%$. Conclusion: there was a high prevalence of sleep disturbances in postmenopausal patients, specially insomnia, apnea and restless legs.

KEYWORDS: Postmenopause; Sleep disorder; Sleep iniciation and maintenance disorders; Sleep apnea syndromes; Restless legs syndrome; Polysomnography

Departamento de Ginecologia - Setor de Climatério, Departamento de Psicobiologia - Medicina e Biologia do Sono - UNIFESP - São Paulo (SP) - Brasil.

1 Professora Adjunta Visitante do Departamento de Psicobiologia - Medicina e Biologia do Sono, responsável pelo ambulatório de Distúrbios do Sono no Climatério do Departamento de Ginecologia - Universidade Federal de São Paulo - UNIFESP - São Paulo (SP) - Brasil.

2 Professora Adjunta do Departamento de Psicobiologia-Medicina e Biologia do Sono - Universidade Federal de São Paulo - UNIFESP - São Paulo (SP) - Brasil.

3 Professor Adjunto do Departamento de Ginecologia - Universidade Federal de São Paulo - UNIFESP - São Paulo (SP) - Brasil.

4 Professor Titular do Departamento de Psicobiologia - Medicina e Biologia do Sono - Universidade Federal de São Paulo - UNIFESP - São Paulo (SP) - Brasil.

5 Professor Titular do Departamento de Ginecologia - Universidade Federal de São Paulo - UNIFESP - São Paulo (SP) - Brasil.

Correspondência: Helena Hachul de Campos

Rua Baltazar Lisboa, 170 - apto 32 - 04110060 - São Paulo - SP - e-mail: helenahachul@psicobio.epm.br; hhachul@ig.com.br - Fax: (011) 2275-9420 


\section{Introdução}

O climatério caracteriza-se por alterações clinicas, metabólicas e regressivas, decorrentes da falência ovariana. Dentre os sintomas mais precocemente observados, sobressaem os vasomotores, que acometem 70 a $80 \%$ das mulheres. Com o agravamento da deficiência estrogênica, sobrevêm alterações da pele e das mamas, dos sistemas neuropsíquico e gênitourinário e do metabolismo ósseo ${ }^{1}$. Além disso, após a menopausa, muitas mulheres passam a apresentar distúrbios do sono ${ }^{2-4}$. Demonstrou-se, por meio de estudo epidemiológico realizado na população de São Paulo, com questionário para avaliação de distúrbios de sono ${ }^{5}$, que $81,6 \%$ dos entrevistados apresentavam pelo menos uma queixa de sono, sendo que $52,1 \%$ dessas estavam relacionadas à insônia ${ }^{6}$.

Sabe-se que as queixas de sono se tornam mais prevalentes com o avançar da idade, sendo 1,5 vezes mais freqüente nos indivíduos com mais de 65 anos $^{7}$. Em um dos maiores estudos populacionais americanos, que avaliou mais de 9000 pacientes, observou-se que cerca de $29 \%$ dos indivíduos com mais de 65 anos tinham dificuldade para manter o sono ${ }^{8}$. Essas queixas foram mais prevalentes em mulheres do que nos homens ${ }^{9}$. Em estudo de base populacional observou-se um aumento relevante da incidência dos distúrbios do sono já na perimenopausa ${ }^{10}$. A menopausa foi considerada fator independente para estes distúrbios após correção dos fatores confundidores como idade, renda ou presença de depressão. Neste estudo, no entanto, o diagnóstico foi baseado apenas em questionário, sem confirmação laboratorial ${ }^{10}$.

A insônia é sintoma que pode ser definido como a dificuldade em iniciar e/ou manter o sono, presença de sono não reparador, ou seja, insuficiente para uma boa qualidade de alerta e bemestar físico e mental durante o dia, com o conseqüente comprometimento do desempenho nas atividades diurnas ${ }^{11}$.

Enquanto a ocorrência de insônia em mulheres com mais de $30 \operatorname{anos}^{8}$ varia de 26 a $45 \%$, estudos epidemiológicos mostraram aumento da sua incidência após a menopausa para cifras entre 28 e $63 \%{ }^{12}$.

Estudos utilizando polissonografia evidenciam que mulheres na pós-menopausa apresentam latência aumentada para o início do $\mathrm{sono}^{3,4} \mathrm{e}$ dificuldade para mantê-lo ${ }^{13-15}$, tendo, portanto, menos eficiência do sono $^{13}$. Não está claro, porém, na literatura, se essas alterações decorrem do estado hormonal alterado nesse período ou do próprio envelhecimento. Além disso, fatores que ocorrem na pós-menopausa, como ondas de calor ${ }^{16-19}$, nictúria ${ }^{19}$ e alterações de humor ${ }^{13,19-21}$, poderiam ter papel mais relevante nos distúrbios do sono do que o estado hormonal per si.

Outro aspecto importante são os distúrbios respiratórios associados ao sono. Em estudo coorte, verificou-se que $83 \%$ das mulheres na pós-menopausa com queixa de insônia tinham, associado, algum grau de distúrbio respiratório do sono $^{22}$. Segundo a Academia Americana de Medicina do Sono (AAMS), a síndrome da apnéia-hipopnéia do sono obstrutiva (SAHSO) caracteriza-se por espisódios recorrentes de obstrução parcial ou total das vias aéreas superiores durante o sono. Manifesta-se como redução (hipopnéia) ou cessação completa (apnéia) do fluxo aéreo, apesar dos esforços inspiratórios. A falta de ventilação alveolar resulta em dessaturação da oxi-hemoglobina e, em casos de eventos prolongados, hipercapnia. Estes eventos são freqüentemente finalizados por despertares abruptos. Sintomas diurnos, como sonolência excessiva, estão relacionados à fragmentação do sono com despertares freqüentes, e possivelmente à hipoxemia recorrente ${ }^{23}$.

Os critérios diagnósticos para a SAHSO, de acordo com a AAMS, incluem sonolência diurna excessiva inexplicável por outros fatores e/ou dois ou mais dos seguintes sintomas e sinais, não explicados por outras condições: asfixia ou respiração difícil durante o sono, despertares noturnos recorrentes, sensação de sono não restaurador, fadiga diurna e dificuldade de concentração. Outro critério é a detecção de cinco ou mais apnéias e/ou hipopnéias e/ou dessaturações relacionadas a esforços respiratórios por hora de sono, obtidos em monitorização durante a noite inteira.

A incidência dessa síndrome diagnosticada pela clínica ou pela polissonografia é de $4 \%$ entre os homens e de $2 \%$ entre as mulheres, predominando em obesos e, entre as mulheres, na pósmenopausa $^{24,25}$.

Outro distúrbio de sono que pode levar à queixa de insônia é o movimento periódico de pernas (MPP). MPP e síndrome das pernas agitadas ou inquietas (SPI) é manifestação clínica em que os pacientes relatam um irresistivel movimento de membros inferiores acompanhado de sensações de "arrastamento" das pernas. Estes sintomas podem levar a despertares noturnos, insônia e irritabilidade. A prevalência de MPP é maior em pacientes idosos: $5 \%$ em indivíduos entre 30 e 50 anos, 29\% entre 51 e 54 anos e $44 \%$ naqueles com idade superior a 65 anos. As queixas de MPP são referidas por $30 \%$ de pessoas anêmicas ou com artrite reumática e por 15 a $20 \%$ de pacientes 
urêmicos ou com predisposição. Está também associado a mielopatias, neuropatias periféricas, diabete, leucemia, artrite reumatóide e fibromiosite e com o uso de drogas antidepressivas tricíclicas, anticonvulsivantes, benzodiazepínicos, barbitúricos e outros hipnóticos.

Há relatos mostrando prevalência de MPP superior a 34\% , especialmente em individuos mais velhos. Não há trabalhos mostrando os efeitos do hipoestrogenismo da pós-menopausa ou dos sintomas climatéricos no MPP. Parece haver alguma associação entre MPP e os esteróides sexuais femininos, já que o MPP aumenta durante a gravidez. Alguns autores ${ }^{25,26}$ mostraram prevalência de 44 a 48\% de MPP em mulheres na pós-menopausa.

Visto que há divergências quanto à ocorrência de distúrbios do sono entre as pós-menopausadas, nosso objetivo foi avaliar a prevalência de distúrbios do sono, tanto subjetiva quanto objetivamente por meio de registro polissonográfico, em amostra bem selecionada de mulheres na pósmenopausa.

\section{Métodos}

Foram selecionadas 33 pacientes que procuraram o atendimento do ambulatório de Climatério da Disciplina de Endocrinologia Ginecológica do Departamento de Ginecologia de Universidade Federal de São Paulo - Escola Paulista de Medicina (UNIFESP-EPM), sem que fossem triadas previamente quanto às queixas de sono. As pacientes assinaram termo de consentimento para participação no estudo, o qual foi aprovado pelo Comitê de Ética em Pesquisa da UNIFESP-EPM.

Foram incluídas no estudo apenas as mulheres com idade entre 50 e 65 anos, que apresentavam no mínimo um ano de amenorréia e niveis de FSH iguais ou superiores a $30 \mathrm{mUI} / \mathrm{mL}$, que não estavam sob tratamento hormonal e que apresentavam exames laboratoriais normais, dentro dos parâmetros para a população estudada.

Foram excluídas do estudo pacientes com diabete melito descompensado, as com infarto do miocárdio recente ou moléstias tromboembólicas, hipertensão arterial grave, insuficiência hepática grave ou em atividade, assim como as que apresentavam eco endometrial superior a $5 \mathrm{~mm}$ à avaliação ultra-sonográfica e/ou teste do progestagênio positivo, ou câncer de endométrio. Foram excluídas as pacientes com hiperplasia atipica de mama prévia ou diagnóstico de câncer de mama. Também foram excluídas as que apresentassem IMC maior ou igual a $30 \mathrm{~kg} / \mathrm{m}^{2}$, ou que estives- sem fazendo uso de medicamentos psicotrópicos durante as três semanas anteriores ao recrutamento para o estudo (antidepressivos, ansiolíticos, benzodiazepínicos, anti-histamínicos e corticosteróides).

Todas as pacientes foram submetidas à rotina propedêutica adotada no Setor de Climatério, que inclui anamnese, exames físico e ginecológico completos e exames subsidiários específicos.

As 33 pacientes selecionadas tinham média de idade de 56 anos, tempo de pós-menopausa médio de 7,7 anos, indice de Kupermann médio de 17 e IMC médio de 27 (Tabela 1).

Tabela 1 - Média, mediana, desvio padrão do índice de Kupperman e de características antropométricas das 33 pacientes estudadas.

\begin{tabular}{lccccccccc}
\hline & $\begin{array}{c}\text { Idade } \\
\text { (anos) }\end{array}$ & Peso & Altura & IMC & \multicolumn{3}{c}{ Cintura quadril } & TP & IK \\
& $(\mathbf{m})$ & $\left(\mathbf{k g} / \mathbf{m}^{2}\right)$ & $(\mathbf{c m})$ & (cm) & (anos) \\
\hline Média & 56 & 65 & 1.55 & 27 & 89 & 99 & 7.7 & 17.0 \\
Mediana & 55 & 66 & 1.55 & 27 & 89 & 98 & 6 & 14.5 \\
DP & 4.5 & 10 & 0.06 & 3.05 & 9.6 & 14 & 6 & 9 \\
\hline IMC = índice de massa corpórea. & & & & & \\
DP = desvio padrão. \\
TP = tempo de pós-menopausa (anos). \\
IK = indice de Kupperman.
\end{tabular}

A avaliação dos distúrbios do sono constou da aplicação de um questionário de sono ${ }^{5}$ e realização de polissonografia. O questionário aplicado ${ }^{5}$ contém perguntas referentes ao sono em geral (quantidade e qualidade), além de questões quanto às queixas relacionadas aos distúrbios do sono. Foi considerado insônia subjetiva quando a paciente se queixava de dificuldade para iniciar e/ou manter o sono e/ou despertar muito antes do horário desejado em pelo menos duas vezes por semana. Foi considerada apnéia subjetiva quando a paciente apresentava queixa de dificuldade respiratória para dormir, sufocação ou relatos do companheiro referindo estes dados em pelo menos uma das sete noites da semana. Foi considerado diagnóstico de pernas inquietas subjetivamente quando a paciente agitava muito as pernas à noite ou pelo relato do companheiro em pelo menos uma das sete noites da semana.

O exame polissonográfico foi realizado no laboratório do sono, com registro de noite inteira, utilizando-se o poligrafo Sleep Analysing Computer SAC Versão 8.1 - (Oxford Instruments Inc.). O exame incluiu eletroencefalograma, eletrooculograma, eletromiograma submentoniano e tibial, eletrocardiograma, registro de fluxo oro-nasal, movimento tóraco-abdominal, registro de ronco, registro de posição corpórea e oximetria. Os exames seguiram o estagiamento do sono proposto por Rechschaffen e Kales $^{27}$. Para análise dos eventos respiratórios fo- 
ram utilizados os padrões de AASM (1999) ${ }^{28}$; para os despertares, os da ASDA (1992) ${ }^{29}$, e para os MPP, os da ASDA $(1999)^{30}$. Foi considerada insônia objetiva quando havia à polissonografia eficiência do sono (tempo de sono dividido pelo tempo de registro de sono) inferior a $85 \%$ e estágio 0 (vigília) superior a $15 \%$. Foi considerada apnéia objetiva como diagnóstico polissonográfico quando o índice de apnéia e hipopnéia (IAH) na polissonografia era maior ou igual a cinco por hora. Foi considerada a ocorrência de pernas inquietas à polissonografia quando se registravam mais que cinco movimentos de pernas por hora.

Para a análise estatística usou-se o cálculo das freqüências de queixas de sono e presença dos distúrbios do sono, em porcentagem, nessa amostra da população.

\section{Resultados}

Observa-se na Figura 1 que a prevalência de insônia subjetiva (queixa) foi de $61 \%$, enquanto a de insônia objetiva foi de $83 \%$. Com relação à apnéia, $23 \%$ das pacientes apresentavam esta queixa e em $27 \%$ dos casos ela foi confirmada pela polissonografia (Figura 2). Já as prevalências subjetiva e objetiva da sindrome de pernas inquietas foram de, respectivamente, 45 e 27\% (Figura 3).

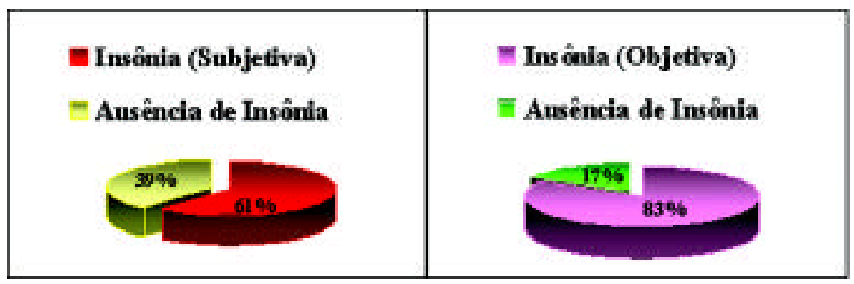

Figura 1 - Prevalência de insônia subjetiva e objetiva (polissonografia) na amostra estudada.

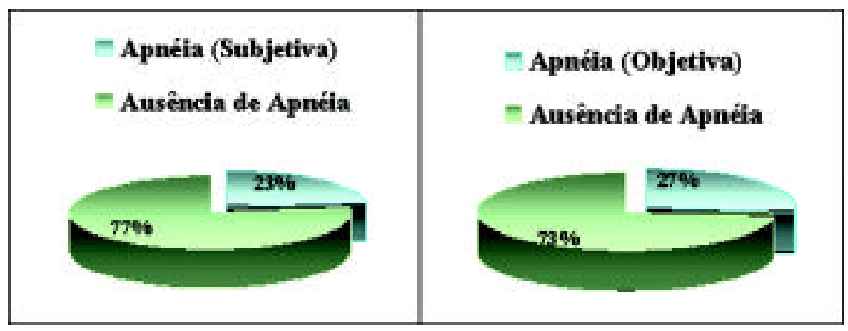

Figura 2 - Prevalência de apnéia subjetiva e objetiva na população estudada.

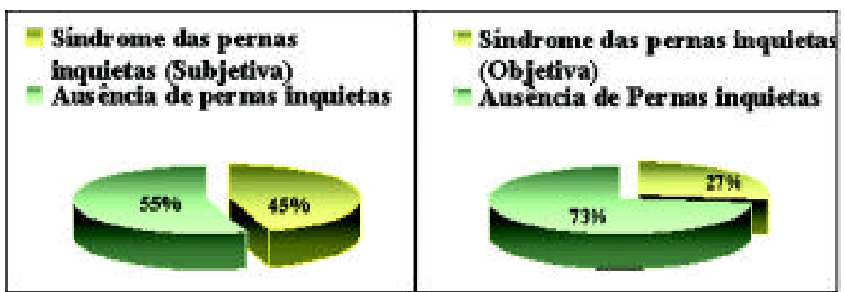

Figura 3 - Prevalência da síndrome das pernas inquietas subjetiva e objetiva na amostra estudada.

\section{Discussão}

Observamos haver alta prevalência de distúrbios do sono na amostra estudada. Essas pacientes, embora tivessem alta taxa de queixa subjetiva $(61 \%)$ e elevada prevalência de insônia na polissonografia (objetiva - $83 \%$ ), somente em $44 \%$ eram coincidentes tanto subjetiva quanto objetivamente. Isso significa que para boa parte das mulheres que referiram insônia não houve confirmação dos diagnósticos à polissonografia e considerável parcela das que não tinham queixa de insônia apresentava, no entanto, polissonografia alterada.

A insônia é o distúrbio de sono mais freqüente na pós-menopausa, em decorrência de alterações hormonais, estados depressivos relacionados a este período da vida, fogachos e/ou à nictúria. Estudos polissonográficos registraram importantes mudanças neste período ${ }^{8}$. No entanto, os resultados destes estudos não conseguem especificar se as alterações no sono eram devidas ao envelhecimento, que por si já alteraria a sua qualidade, ou ao estado menopausal. De qualquer forma, verifica-se menor eficiência do sono na pósmenopausa ${ }^{13}$. As mulheres nessa fase apresentam maior latência para o sono ${ }^{3,4}$, dificuldade de manutenção do sono e, portanto, mais insônia ${ }^{14,15}$ quando comparadas àquelas na pré-menopausa. Shaver e Zenk ${ }^{16}$ relatam que os distúrbios do sono no climatério estão relacionados a sintomas como ondas de calor e transpiração, os quais ocorrem em geral simultaneamente.

A caracterização da transição menopausal fundamenta-se nas alterações do ciclo menstrual, na presença de ondas de calor e níveis elevados de FSH. Já foi relatado que mulheres com fogachos apresentam aumento do estágio 4 e diminuição da eficiência do sono e dos períodos de REM, comparativamente às mulheres sem fogachos ${ }^{16}$. Tais fatos sugerem que o estado hormonal teria influência na qualidade do sono. Não está claro, entretanto, se esta alteração se deve aos fogachos ou se a instabilidade hormonal ocasionaria os fogachos e a alteração no sono simultaneamente ${ }^{18}$. Aparentemente há correlação entre a ocorrência de fogachos e de despertares em mulheres na pós-menopausa, o que não foi detectado na pré-menopausa ${ }^{18}$. Neste estudo, oito entre nove pacientes apresentaram essa correlação. No entanto, foram incluídas somente pacientes com fogachos acentuados, e o número de casos foi pequeno. Nesta série, a terapia com 50 microgramas de etinilestradiol, 4 vezes ao dia, por 30 dias, melhorou significativamente tanto as queixas de fogachos como a de insônia. 
Por outro lado, vários autores assinalaram que a nictúria pode levar a distúrbios do sono na pós-menopausa com maior freqüência do que os sintomas vasomotore ${ }^{20}$. Outros estudos sugerem que as mudanças de humor que se verificam na pós-menopausa seriam resultantes de distúrbios no sono decorrentes dos fogachos que ocorrem durante esse período ${ }^{19,21}$. Os distúrbios do sono e as alterações de humor em mulheres na peri e na pré-menopausa foram analisados por alguns autores ${ }^{12}$ por meio de questionários subjetivos e monitor actinográfico. Observou-se, na perimenopausa, maior número de despertares durante a noite e por períodos maiores, os quais estavam associados aos altos niveis de ansiedade (alterações de humor). Verificou-se, em um estudo longitudinal com 213 mulheres na pós-menopausa, aumento dos distúrbios do sono, quando as pacientes não estavam submetidas a terapia hormonal ${ }^{31}$.

Em nosso estudo, o número de pacientes que se queixou de apnéia (23\%) foi semelhante ao que apresentou apnéia no registro polissonográfico $(27 \%)$. A alta prevalência de apnéia estaria relacionada, possivelmente, à deficiência hormonal, à distribuição de gordura de modo mais androgênica após a menopausa e à proporção de gordura no pescoço.

Registramos, também, que o número de pacientes que referiu pernas inquietas $(45 \%)$ também foi superior ao encontrado nos registros polissonográficos $(27 \%)$. Sabe-se que a incidência de pernas inquietas aumenta com a idade. Assim, haveria para alguns autores alta incidência de pernas inquietas após a menopausa, tanto em $\mathrm{mu}-$ lheres sob estrogenioterapia, como nas que estavam usando placebo ${ }^{20}$. Embora se considere que a incidência de pernas inquietas deva sofrer alguma influência hormonal, já que aumenta na gravidez, os estudos nesta área são ainda escassos.

As divergências observadas entre a avaliação subjetiva e os resultados obtidos com o emprego da polissonografia foram relatados em outros estudos ${ }^{32}$. Esta falta de correlação entre os dois métodos indica que ambos devem ser empregados em associação para se obter avaliação adequada da prevalência dos vários distúrbios do sono.

Nossa população de estudo incluiu mulheres na pós-menopausa que procuravam acompanhamento em ambulatório de climatério, sem seleção prévia das que tivessem queixas de distúrbios do sono. Utilizamos método bem estabelecido para estudar as alterações do sono, ou seja, questionário específico e polissonografia, e usamos critérios rígidos para caracterizar a insônia, a apnéia e os movimentos periódicos de pernas pela polissonografia. Nossos resultados mostraram que, na pós-menopausa, ocorre maior prevalência de distúrbios do sono, como insônia e apnéia, com piora da qualidade do sono. Desse modo, por constituir importante problema de saúde pública, os distúrbios do sono devem ser bem avaliados, tratados e acompanhados.

\section{Referências}

1. Baracat EC, Simões MJ, Soares JM Jr, Haidar MA, Rodrigues de Lima G. Ultrastructural aspects of the postmenopausal endometrium after oral or transdermal estrogen administration. Clin Exp Obstet Gynecol. 2001;28(1):26-30.

2. von Muhlen DG, Kritz-Silverstein D, BarrettConnor. A community-based study of menopause symptoms and estrogen replacement in older women. Maturitas. 1995;22(2):71-8.

3. McKinlay SM, Jefferys M. The menopausal syndrome. Br J Prev Soc Med. 1974;28(2):108-15.

4. Dennerstein L, Smith AM, Morse C, Burger H, Green A, Hopper J, et al. Menopausal symptoms in Australian women. Med J Aust. 1993;159(4):232-6.

5. Braz S, Neumann BRG, Tufik S. Avaliação dos distúrbios do sono: elaboração e validação de um questionário. Rev ABP-APAL. 1987;9(1):9-14.

6. Tufik S, Nery LE, Bittencourt LR, Palombini L, Bagnato M, Moura SM, et al. Distúrbios do sono. RBM Rev Bras Med. 1997;54(1/2):12-30.

7. Mellinger GD, Balter MB, Uhlenhuth EH. Insomnia and its treatment. Prevalence and correlates. Arch Gen Psychiatry. 1985;42(3):225-32.

8. Carskadon MA, Dement WC, Mitler MM, Guilleminault C, Zarcone VP, Spiegel R. Self-reports versus sleep laboratory findings in 122 drug-free subjects with complaints of chronic insomnia. Am J Psychiatry. 1976;133(12):1382-8.

9. Bliwise DL, King AC, Harris RB, Haskell WL. Prevalence of self-reported poor sleep in healthy population aged 50-65. Soc Sci Med. 1992;34(1):49-55.

10. Shin C, Lee S, Lee T, Shin K, Yi H, Kimm K, et al. Prevalence of insomnia and its relationship to menopausal status in middle-aged Korean women. Psychiatry Clin Neurosci. 2005;59(4):395-402.

11. Poyares D, Aloe F, Rizzo GNV, Minhoto G, Pinto Junior LR, Bezerra MLS, et al. I Consenso Brasileiro de Insônia. Hypnos. 2003;4(Supl 2):5-39.

12.Lugaresi E, Cirignotta F, Zucconi M. Good and poor sleepers: an epidemiological survey of San Marino population. In: Guilleminault C, Lugaresi E, editors. Sleep/wake disorders: natural history, epidemiology, and longterm evolution. New York: Raven Press; 1983. p. 1-12. 
13. Baker A, Simpson S, Dawson D. Sleep disruption and mood changes associated with menopause. J Psychosom Res. 1997;43(4):359-69.

14. Ballinger CB. Subjective sleep disturbance at the menopause. J Psychosom Res. 1976;20(5):509-13.

15. Campos HH, Bittencourt LRA, Haidar MA, Tufik S, Baracat EC. Distúrbios do sono no climatério. Femina. 2005;33(11):815-20.

16.Shaver JL, Zenk SN. Sleep disturbance in menopause. J Womens Health Gend Based Med. 2000;9(2):109-18.

17. Woodward S Freedman RR. The thermoregulatory effects of menopausal hot flashes on sleep. Sleep. 1994;17(6):497-501.

18. Manber R, Armitage R. Sex steriods, and sleep: a review. Sleep. 1999;22(5):540-55.

19. Erlik Y, Tataryn IV, Meldrum DR, Lomax P, Bajorek JG, Judd HL. Association of waking episodes with menopausal hot flushes. JAMA. 1981;245(17):17414.

20.Polo-Kantola P, Erkkola R, Helenius H, Irjala K, Polo O. When does estrogen replacement therapy improve sleep quality? Am J Obstet Gynecol. 1998;178(5):1002-9.

21. Freedman RR, Woodward S, Mayes MM. Nonneural mediation of digital vasodilation during menopausal hot flushes. Gynecol Obstet Invest. 1994;38(3):2069.

22. Guilleminault C, Palombini L, Poyares D, Chowdhuri S. Chronic insomnia, postmenopausal women, and sleep disordered breathing: part 1. Frequency of sleep disordered breathing in a cohort. J Psychosom Res. 2002;53(1):611-5.

23. Bittencourt LRA. Tratamento clínico e cirúrgico da síndrome da apnéia e hipopnéia obstrutiva do sono (SAHOS). Rev Bras Med. 2001;58(8):618-30.
24. Bixler EO, Vgontzas AN, Lin HM, Ten Have T, Rein J, Vela-Bueno A, et al. Prevalence of sleepdisordered breathing in women: effects of gender. Am J Respir Crit Care Med. 2001;163(3 Pt1):608-13.

25. Young T, Finn L, Austin D, Peterson A. Menopausal status and sleep-disordered breathing in the Wisconsin Sleep Cohort Study. Am J Respir Crit Care Med. 2003;167(9):1181-5.

26. Polo-Kantola P, Rauhala E, Erkkola R, Irjala K, Polo O. Estrogen replacement therapy and nocturnal periodic limb movements: a randomized controlled trial. Obstet Gynecol. 2001;97(4):548-54.

27. Rechschaffen A, Kales A. A manual of standardized terminology, techniques and scoring system for sleep stages of human subjects. Los Angeles: UCLA Brain Information Service/Brain Research Institute; 1968.

28. Sleep-related breathing disorders in adults: recommendations for syndrome definitions and measurement techniques in clinical research. The Report of an American Academy of Sleep Medicine Task Force. Sleep. 1999;22(5):667-89.

29.EEG arousals: scoring rules and examples: a preliminary report from the Sleep Disorders Atlas Task Force of the American Sleep Disorders Association. Sleep. 1992;15(2):173-84.

30. Chesson AL Jr, Wise M, Davila D, Johnson S, Littner M, Anderson WM, et al. Practice parameters for the treatment of restless legs syndrome and periodic limb movement disorder. An American Academy of Sleep Medicine Report. Standards of Practice Committee of the American Academy of Sleep Medicine. Sleep. 1999;22(7):961-8.

31. Owens JF Matthews KA. Sleep disturbance in healthy middle-aged woman. Maturitas. 1998;30(1):41-50.

32. Weaver EM, Kapur V, Yueh B. Polysomnography vs self-reported measures in patients with sleep apnea. Arch Otolaryngol Head Neck Surg. 2004;130(4):453-8. 